\title{
Review of: "Comparison of oral metabolome profiles of stimulated saliva, unstimulated saliva, and mouth- rinsed water"
}

Ramesh Nagarajappa

Potential competing interests: The author(s) declared that no potential competing interests exist.

The article is on comparison of oral metabolome profiles of stimulated saliva, unstimulated saliva, and mouth-rinsed water.

The study is done and written well. No significant comments from my end. 